\title{
Coleção de tipos de Plecoptera (Insecta) do Museu Nacional (UFRJ), Rio de Janeiro, Brasil
}

\author{
Fernanda Avelino Capistrano da Silva ${ }^{1,2,3}$ \& Janira Martins Costa ${ }^{2}$

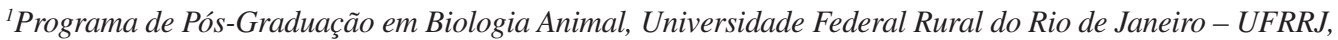 \\ BR-465, Km 7, CEP 23890-000, Seropédica, RJ, Brazil, \\ ${ }^{2}$ Laboratório de Insetos Aquáticos, Museu Nacional, Universidade Federal do Rio de Janeiro - UFRJ, \\ Quinta da Boa Vista, São Cristóvão, CEP 20940-040, Rio de Janeiro, RJ, Brazil \\ ${ }^{3}$ Autor para correspondência: Fernanda Avelino Capistrano da Silva, e-mail: fernandaacsilva@yahoo.com.br
}

AVELINO-CAPISTRANO, F.S. \& COSTA, J.M. Types of Plecoptera (Insecta) in the collection of the National Museum (UFRJ), Rio de Janeiro, Brazil. Biota Neotrop., 9(4): http://www.biotaneotropica.org.br/v9n4/en/ abstract?article+bn01809042009.

Abstract: Types of Plecoptera (Insecta) in the collection of the National Museum (UFRJ), Rio de Janeiro, Brazil. The Insect Type Collection of National Museum contains 3,263 specimens of which 57 are in the Order Plecoptera (2 holotypes, 1 allotypes, 54 paratypes), belonging to families Gripopterigydae and Perlidae. The state of preservation of types and geographical coordinates of the collection localities are provided.

Keywords: neotropical stoneflies, biodiversity, collection conservation, inventory.

AVELinO-CAPISTRAnO, F.S. \& COSTA, J.M. Coleção de tipos de Plecoptera (Insecta) do Museu Nacional (UFRJ), Rio de Janeiro, Brasil. Biota Neotrop., 9(4): http://www.biotaneotropica.org.br/v9n4/pt/ abstract?article+bn01809042009.

Resumo: Coleção de Tipos de Plecoptera (Insecta) do Museu Nacional (UFRJ), Rio de Janeiro, Brasil. A Coleção de Tipos de Insetos do Museu Nacional inclui 3.263 espécimes dos quais 57 são da ordem Plecoptera (2 holótipos, 1 alótipos e 54 parátipos), pertencentes às famílias Gripopterygidae e Perlidae. O estado de preservação dos tipos e as coordenadas geográficas das localidades de coleta são fornecidas.

Palavras-chave: plecópteros neotropicais, biodiversidade, conservação de coleções, inventário. 


\section{Introdução}

Coleção taxonômica é a reunião ordenada de espécimes, devidamente preservados, sendo fonte imprescindível para estudos taxonômicos e de grande importância para o conhecimento da Biodiversidade. A coleção entomológica do Museu Nacional (UFRJ) conta com aproximadamente seis milhões de exemplares, distribuídos em diferentes ordens. Deste total, 3.263 são de material Tipo.

A Coleção de Insetos Aquáticos do Museu Nacional possui cerca de 500 mil espécimes, dentre eles incluí-se os Plecoptera com aproximadamente 1.200 exemplares, provenientes de diferentes localidades. Os primeiros espécimes de Plecoptera coligidos à Coleção entomológica do Museu Nacional datam de 1957, compostos em sua maior parte, de adultos, coletados no Estado do Rio de Janeiro pelo Dr. Newton Dias dos Santos, ex-pesquisador do Museu Nacional. Os tipos depositados nesta coleção são oriundos de pesquisas realizadas no Brasil pelos pesquisadores Stanley G. Jewett Jr, em 1960, e Claudio G. Froehlich, entre 1990-1994.

No Brasil, a ordem Plecoptera engloba duas famílias: Gripopterygidae com quatro gêneros e 36 espécies e, Perlidae com quatro gêneros e aproximadamente 100 espécies (Lecci \& Froehlich, 2007).

\section{Material e Métodos}

O material examinado inclui imaturos e adultos depositados na Coleção de Insetos Aquáticos do Museu Nacional. Os exemplares encontram-se fixados em álcool a 70\%, acondicionados em tubo de ensaio, contendo em seu interior a etiqueta original ou uma nova etiqueta caso a original encontre-se em más condições, tendo-se o cuidado de transcrever todas as informações originais fornecidas pelos coletores.

Os nomes estão listados sob cada família e subfamília, seguidos pelo nome do autor, data e página da fonte original (periódico ou livro) onde a descrição da espécie foi publicada. Citações subsequentes à

Tabela 1. Siglas e nome dos coletores.

Table 1. Acronyms and names of collectors.

\begin{tabular}{ll}
\hline \multicolumn{1}{c}{ Sigla } & \multicolumn{1}{c}{ Nome } \\
\hline A.R. Barros & Alfredo Rego Barros \\
C.G. Froehlich & Claudio Gilberto Froehlich \\
D. Albuquerque & Dalcy Albuquerque \\
G.M. Cavasin & Gláucia Maria Cavasin \\
J.P. Machado & Joaquim Pereira Machado \\
L.G. Oliveira & Leandro Gonçalves Oliveira \\
M.Chapot-Preveaust & Myrian Chapot-Preveaust \\
M.J.N.Ferreira & Maria José Nascimento Ferreira \\
M.Schweiger & Monica Schweiger \\
N.D. Santos & Newton Dias dos Santos \\
G. Shimizu & Gisela Yuka Shimizu \\
\hline
\end{tabular}

descrição original da espécie são fornecidas como referências adicionais; breve comentário para cada táxon com informações sobre a situação do material tipo é fornecido.

Siglas, abreviaturas e símbolos utilizados no texto: (Col.) coletor; (Cols.) coletores; (s.c.) sem coletor; (*) exemplar não examinado; para os nomes dos coletores, verificar Tabela 1. Para os locais de coleta do material tipo, utilizaram-se as coordenadas geográficas de acordo com o Site Global Gazetteer (http://www.fallingrain.com/world/). (Tabela 2).

\section{Lista dos tipos}

\section{GRIPOPTERYGIDAE}

Gripopteryx Pictet, 1841

Gripopteryx coruja Froehlich, 1993

Gripopteryx coruja Froehlich, 1993: 25-29, fig.3-9; Heckman, 2003: 265-266, fig. 5.340.

Parátipos - Brasil, Estado de São Paulo, São Paulo, Reserva Casa Grande, Ribeiro da Coruja, col. C.G. Froehlich, 13.X.1973, 1 ninfa (n ${ }^{\circ}$ 137); Salesópolis, Estação Biológica de Boracéia (Pedreira), col. C.G. Froehlich, 25.IX.1987, 1 ninfa ( $\left.{ }^{\circ} 157\right)$; mesma localidade, Cols.: C.G. Froehlich \& M.Schweiger., 05-22. XII.1987, 1 \& (n $\left.{ }^{\circ} 159\right)$.

Comentários: Exemplares acondicionados em álcool 70\%, em bom estado.

Gripopteryx juetah Froehlich, 1990

Gripopteryx juetah Froehlich 1990: 242-245, fig.21-33; Heckman, 2003: 263, fig. 5.336.

Parátipos - Brasil, Estado de São Paulo, Campos do Jordão, Parque Estadual de Campos do Jordão, Córrego Casquilho, Col. C.G. Froehlich., 05.III.1986, 5 ninfas ( $\left.\mathrm{n}^{\circ} 150\right)$; mesma localidade, Córrego do Campo do Meio, Col. C.G. Froehlich \& L.G. Oliveira, 08.V.1986, 5 ninfas (n 151); Minas Gerais, Sapucaí-Mirim, Sítio São Miguel (Serra), Col. L.G. Oliveira, 06.IX.1986, 3 $\widehat{\jmath}, 1$ q(nº 152).

Comentários: Exemplares acondicionados em álcool 70\%, em bom estado. Ninfas de diversos instares.

Gripopteryx liana Froehclich, 1993

Gripopteryx liana Froehclich, 1993: 21-34, figs.17-25; Heckman, 2003: 262-263, fig. 5.335.

Parátipos - Brasil, Estado de Minas Gerais, Serra do Cipó, Chapéu do Sol, (km 110, na estrada para Conceição do Mato Dentro), Col. C.G. Froehlich, I. Carvalho \& Shimizu, 21.IV.1975, $1 \widehat{\jmath}\left(\mathrm{n}^{\circ} 139\right)$; mesma localidade (km 126), col. C.G. Froehlich, 24.IX.1976, 1 ninfa ( $\left.\mathrm{n}^{\circ} 145\right)$.

Comentários: Exemplares acondicionados em álcool 70\%, em bom estado.

Tabela 2. Coordenadas geográficas das localidades de coleta.

Table 2. Geographical coordinates of the collection locality.

\begin{tabular}{|c|c|c|c|c|}
\hline Estado & Cidade & Latitude & Longitude & Altitude (m) \\
\hline Espírito Santo & Santa Teresa, Santa Lucia, Reserva do Museu Nacional & $19^{\circ} 55^{\prime} 0 ” \mathrm{~S}$ & $40^{\circ} 36^{\prime} 0 ” \mathrm{~W}$ & 900 \\
\hline \multirow[t]{2}{*}{ Minas Gerais } & Jaboticatubas, Serra do Cipó & $19^{\circ} 30^{\prime} 0 ” \mathrm{~S}$ & $43^{\circ} 45^{\prime} 0^{\prime \prime} \mathrm{W}$ & 900 \\
\hline & Sapucaí-Mirim, Sítio São Miguel & $22^{\circ} 43^{\prime} 60^{\prime \prime} \mathrm{S}$ & $45^{\circ} 45^{\prime} 0 ” \mathrm{~W}$ & 927 \\
\hline \multirow[t]{3}{*}{ Rio de Janeiro } & Teresópolis, Bom Retiro & $22^{\circ} 25^{\prime} 34^{\prime \prime} \mathrm{S}$ & $42^{\circ} 57^{\prime} 28^{\prime \prime} \mathrm{W}$ & 910 \\
\hline & Petrópolis & $22^{\circ} 30^{\prime} 39^{\prime \prime} \mathrm{S}$ & $43^{\circ} 11^{\prime} 4 " \mathrm{~W}$ & 838 \\
\hline & Petrópolis, Alto da Mosela & & & 1.200 \\
\hline \multirow[t]{2}{*}{ São Paulo } & Salesópolis & $23^{\circ} 31^{\prime} 55^{\prime \prime} \mathrm{S}$ & $45^{\circ} 50^{\prime} 51^{\prime \prime} \mathrm{W}$ & 780 \\
\hline & Campos do Jordão & $22^{\circ} 43^{\prime} 60^{\prime \prime} \mathrm{S}$ & $45^{\circ} 34^{\prime} 60^{\prime \prime} \mathrm{W}$ & 1736 \\
\hline
\end{tabular}


Gripopteryx maculosa Jewett, 1960

Gripopteryx maculosa Jewett 1960: 171, figs.2, 2A-C; Froehlich, 1993:24-25, Fig.2; Heckman, 2003: 264-265, fig. 5.338.

Holótipo - Brasil, Estado do Rio de Janeiro, Petrópolis, Le Vallon, Alto Mosela, Col. D. Albuquerque \& J.P. Machado, III.1957, 1 ô (nº 96)*.

Alótipo - Estado do Espírito Santo, Santa Teresa, Reserva do Museu Nacional Santa Lucia ${ }_{s}$ Cols. N.D. Santos, A.R. Barros \& J.P. Machado, 18.XI.1965, 1 \& ( $\left.{ }^{\circ} 100\right)^{*}$.

Parátipos - Brasil, Estado do Rio de Janeiro, Petrópolis, Le Vallon, Alto Mosela, Cols. D. Albuquerque \& J.P. Machado, III.1957, 1 ऽ (ñ 101); mesma localidade, II-III.1958, Cols. D. Albuquerque \& J.P. Machado, $5 \delta^{\top *}\left(n^{\circ} 95\right)$.

Comentários: O parátipo de número $\mathrm{n}^{\circ} 101$ encontra-se em bom estado de conservação, abdômen diafanizado.

Gripopteryx pilosa Froehlich, 1990

Gripopteryx pilosa Froehlich 1990: 245-247, figs. 34-43; Heckman, 2003: 259, fig. 5.294.

Parátipos - Brasil, Estado de São Paulo, Campos do Jordão, Parque Estadual de Campos do Jordão, Córrego Galharada, Col. C.G. Froehlich, 11.XI.1985, 7 ninfas ( $\left.{ }^{\circ} 149\right)$; mesma localidade, Col. C.G. Froehlich, 20.I.1987, 1 q +1 exúvia (nº 155); mesma localidade, Col. C.G. Froehlich, 20.XI.1987, 5 ninfas ( $\left.n^{\circ} 158\right)$.

Comentários: Exemplares acondicionados em álcool 70\%, em bom estado; ninfas de diversos instares.

Gripopteryx pinima Froehlich, 1993

Gripopteryx pinima Froehlich, 1993: 29-31, figs. 10-16; Heckman, 2003: 265-266, fig. 5.339.

Parátipos - Brasil, Estado de São Paulo, Salesópolis, Estação Biológica de Boracéia (850 m), Col. C.G. Froehlich, 24-30.I.1979, 1 + +1 exúvia ( $\left.n^{\circ} 426\right)$; mesma localidade, Col. C.G. Froehlich, 05.III.1989, 1 ô ( $n^{\circ}$ 165); mesma localidade, Col. C.G. Froehlich, 28.I.1983, 4 ninfas ( $\mathrm{n}^{\circ}$ 148).

Comentários: Exemplares acondicionados em álcool 70\%, em bom estado.

Paragripopteryx Enderlein, 1909

Paragripopteryx delicata Froehlich, 1994

Paragripopteryx delicata Froehlich, 1994:229-231, figs.1-5; Heckman, 2003: 279, fig. 5.360.

Parátipos - Estado de São Paulo, Estação Biológica Boracéia, Ribeiro Veneranda, s.c., 02.XI.1975. 1 ð (nº 138); mesma localidade, Col. C.G. Froehlich, 11-12.VIII.1988, 3 ninfas ( $n^{\circ} 161$ ); Campos do Jordão, Parque Estadual Córrego Galharada (1.600 m), Col. C.G. Froehlich, 30.X.1986, 1 q ( $\left.{ }^{\circ} 154\right)$; mesma localidade, Col. C.G. Froehlich \& L.G. Oliveira, 16.XII.1987, $1 \hat{\jmath}\left(n^{\circ} 160\right)$.

Comentários - Exemplares acondicionados em álcool 70\%, em bom estado. Ninfas de diversos instares.
Paragripopteryx guardae Froehlich, 1994

Paragripopteryx guardae Froehlich, 1994:231-332, figs. 6-8; Heckman, 2003: 277, fig. 5.357.

Parátipo - Estado de São Paulo, Campos do Jordão, Parque Estadual, Córrego Campo do Meio (1.600 m), Cols. C.G. Froehlich \& L.G. Oliveira, 03.X.1986, 1 đิ + exúvia (nº 153); mesma localidade, Cols. C.G. Froehlich, L.G. Oliveira, M.J.N. F \& G.M. Cavasin, 17-20.XI.1987, 2 ( n $\left.^{\circ} 156\right)$.

Comentários: exemplares acondicionados em álcool 70\%, em bom estado.

\section{PERLIDAE}

Anacroneuria Klapálek, 1909

Anacroneuria furfurosa Jewett, 1960

Anacroneuria furfurosa Jewett 1960:173-174, figs.9, 9A; Heckman, 2003: 114-115, fig. 5.135.

Holótipo - Estado do Rio de Janeiro, Bom Retiro, Col. N.D. Santos, J.P. Machado \& A.R. Barros, 08.I.1957, 1 q.(n $\left.{ }^{\circ} 93\right)$.

Paratipo - Estado do Rio de Janeiro, Bom Retiro, Col. N.D. Santos, J.P. Machado \& A.R. Barros, 08.I.1957, 1 q (nº 94).

Comentários: Holótipo com o $2^{\circ}$ e $3^{\circ}$ pares de pernas quebrados, abdômen diafanizado; Alótipo com as asas amassadas e todas as pernas quebradas.

\section{Agradecimentos}

Ao Prof. Dr. Cláudio G. Froehlich (USP-Ribeirão Preto) e a Prof ${ }^{a}$. Dr $^{\mathrm{a}}$.Tatiana Chrysostomo Santos (MN_UFRJ) pela leitura crítica deste manuscrito. À CAPES pela bolsa de Mestrado, CNPQ pela bolsa de produtividade e a FAPERJ pelo apoio financeiro à conservação do acervo da Coleção de Insetos Aquáticos.

\section{Referências Bibliográficas}

ENDERLEIN, G. 1909. Klassifikation der Plecopteren, sowie Diagnosen neuer Gattungen und Arten. Zool. Anz. 34:385-419.

HECKMAN, C.W. 2003. Encyclopedia of South American Aquatic Insects: plecoptera. Kluwer Academic Publishers, Dordrecht, 329p.

KLAPÁLEK, F.R. 1909.Vorläufiger Bericht über exotische Plecopteren. Wiener Ent. Ztg. 28:215-232.

FROEHLICH, C.G. 1990. Brazilian Plecoptera 6. Gripopteryx from Campos do Jordão, State of São Paulo (Gripopterygidae). Stud. Neotrop. Fauna \& Environm. 25(4):235-247.

FROEHLICH, C.G. 1993. Brazilian Plecoptera 6. Old and new species of Gripopteryx (Gripopterygidae). Aquatic Insects, 15(1):21-38.

FROEHLICH, C.G. 1994. Brazilian Plecoptera 8. On Paragripopteryx (Gripopterygidae), Aquatic Insects, 16(4):227-339.

JEWETT, S.G.J. 1960. Notes and descriptions concerning Brazilian stoneflies. Arq. Mus. Nac., 1: 167-183.

LECCI, L.S. \& FROEHLICH, C.G. 2007. Plecoptera. In Guia on-line: identificação de larvas de Insetos Aquáticos do Estado de São Paulo (C.G Froehlich, org.). http://sites.ffclrp.usp.br/aguadoce/guiaonline.

PICTET, F. 1841. Histoire naturelle générale et particulière des insectes Névroptères. Famille des Perlides. Kessmann, Geneva, p.1-423.

Recebido em 21/05/09

Versão reformulada recebida em 29/07/09

Publicado em 06/11/09 